\title{
Aplicación del método MOORA para la gestión de la carga de trabajo en la atención de pacientes con COVID-19
}

\author{
Volumen XXI No (2). Julio-Diciembre. Pág. 111-120
}

ISSN: 0121-1048 IMPRESO ISSN: 2422-3220 EN LÍNEA

\author{
López Castañeda, Jesús \\ Raymundo \\ Universidad Autónoma de Ciudad \\ Juárez, Ciudad Juárez, \\ Chih.Departamento de Ingeniería \\ Industrial y de Sistemas, México \\ al159621@alumnos.uacj.mx \\ ORCID: 0000-0003-0507-5418
}

\section{Pérez-Domínguez, Luis Asunción}

Universidad Autónoma de Ciudad Juárez, Ciudad Juárez,

Chih.Departamento de Ingeniería Industrial y de Sistemas, México, luis.dominguez@uacj.mx

ORCID: 0000-0003-2541-4595

\author{
García Alcaraz, Jorge Luis \\ Universidad Autónoma de \\ Ciudad Juárez, Ciudad Juárez, \\ Chih.Departamento de \\ Ingeniería Industrial y de \\ Sistemas, México, \\ jorge.garcia@uacj.mx
}

ORCID: 0000-0002-7092-6963

\begin{abstract}
Resumen
La pandemia por COVID-19 fue difícil para todos y aún más para los hospitales y el personal médico. Los contagios por esta enfermedad fueron muchos en muy poco tiempo debido a esto los hospitales recurrieron a utilizar herramientas de ingeniería para gestión eficaz y eficiente de los pacientes con síntomas graves de COVID-19. Considerando lo anterior es posible aplicar un método de decisión multicriterio para priorizar los objetivos que se requieren cumplir para lograr la mejor atención medica de los pacientes con síntomas graves. Los resultados obtenidos es que el criterio que se debe enfocar primero es la utilización de plataformas digitales para la comunicación como WeChat, WhatsApp, Telegram, debido a que dichas plataformas ofrecen una comunicación instantánea por medio de texto, imágenes, documentos, videos o audios y que no requiere presencia física entre la sala de aislamiento y el exterior, en comparación con los obtenidos en la Universidad de Huazhong, este método definiría el orden de prioridad en que se deben realizar estos objetivos. Finalmente, los resultados obtenidos demuestran que es posible establecer prioridad en la gestión de las cargas de trabajo en la atención de los pacientes con la enfermedad COVID.
\end{abstract}

Palabras claves: MOORA, Escala de Likert, PDCA, COVID-19.

Códigos JEL: D14, D61, I31, O33, 035, P18.

Recibido: 04/04/2021. Aceptado: 26/07/2021. Publicado: 18/12/2021.

\footnotetext{
• Citación: López Castañeda, J. R., Pérez-Domínguez, L. \& García Alcaraz, J. L. (2021). Aplicación del método MOORA para la gestión de la carga de trabajo en la atención de pacientes con COVID-19. Inquietud Empresarial, 21(1), 111-120. https://doi.org/10.19053/01211048.12555
} 
Aplicación del metodo moora para la gestión de la carga de trabajo en la atención de pacientes

con COVID-19

\title{
Application of the MOORA method for the management of the workload in the care of patients with COVID-19
}

\begin{abstract}
The COVID-19 pandemic was difficult for everyone and even more so for hospitals and medical personnel. The infections caused by this disease were many in a very short time, due to this, hospitals resorted to using engineering tools for effective and efficient management of patients with severe symptoms of COVID-19. Considering the above, it is possible to apply a multi-criteria decision method to prioritize the objectives that must be met to achieve the best medical care for patients with severe symptoms. The results obtained is that the criterion that should be approached first is the use of digital communication platforms such as WeChat, Whatsapp, Telegram, because these platforms offer instant communication through text, images, documents, videos or audios and which does not require physical presence between the isolation room and the outside, compared to those obtained at Huazhong University, this method would define the order of priority in which these objectives should be achieved. Finally, the results obtained depict that it is possible to prioritize the management of workloads in the care of patients with COVID disease.
\end{abstract}

Keywords MOORA, Likert scale, PDCA, COVID-19.

JEL codes: D14, D61, I31, O33, O35, P18.

\section{INTRODUCCIÓN}

El año pasado fue difícil para todo el mundo debido a la pandemia por el coronavirus $y$, aun mas difícil fue para los hospitales y el personal médico de todo el mundo que tuvieron que lidiar con una enfermedad de la cual era desconocida y que había logrado contagiar a muchas personas en poco tiempo. Los hospitales para atender de manera eficiente y eficaz a la gran cantidad de pacientes con síntomas graves empezaron a recurrir a herramientas de ingeniera como el ciclo PDCA (Plan, Do, Check and Action). Dicho ciclo tiene diversas aplicaciones una aplicación resaltable es la de la Facultad de Medicina de Tongji de la Universidad de Huazhong en Wuhan, China que aplicaron dicho ciclo para la gestión de pacientes de síntomas críticos de COVID-19. Con la información que nos proporciona el artículo de Li (2020) es posible implementar un método multicriterio como el método MOORA (Método Multi Objetivo de Optimización basado en Análisis de Radio), utilizando el método antes dicho lo que haremos es utilizar los resultados obtenidos del ciclo PDCA he implementarlos en la secuencia de pasos del método MOORA.

El documento está organizado de tal manera que en la Sección 2 se presentan los conceptos preliminares del artículo, en la Sección 3 se describe la metodología utilizada,

\section{CONCEPTOS PRELIMINARES}

En esta sección se explican los conceptos básicos necesarios para el correcto entendimiento del artículo. 


\subsection{Ciclo PDCA}

El ciclo PDCA (Plan, Do, Check and Action) es una herramienta para la mejora continua de un proceso que enseña a las empresas $u$ organizaciones planificar una acción (Johnson, 2002). Dicho ciclo está compuesto por cuatro etapas:

$\rightarrow$ Planear: Se buscan las actividades en el sistema que se pueden mejorar y se establecen los objetivos a alcanzar.

$\rightarrow$ Hacer: Se realizan los cambios propuestos en el sistema.

$\rightarrow$ Revisar: Una vez implementada la mejora, se verifica el correcto funcionamiento de esta definiendo un periodo de prueba.

$\rightarrow$ Actuar: Una vez terminado el periodo de prueba se analizan los resultados realizando una comparación entre el antes y el después de la mejora. Para luego determinar si implementar o no la mejora propuesta generando una retroalimentación para futuras mejoras.

FIGURA 1. CICLO PDCA

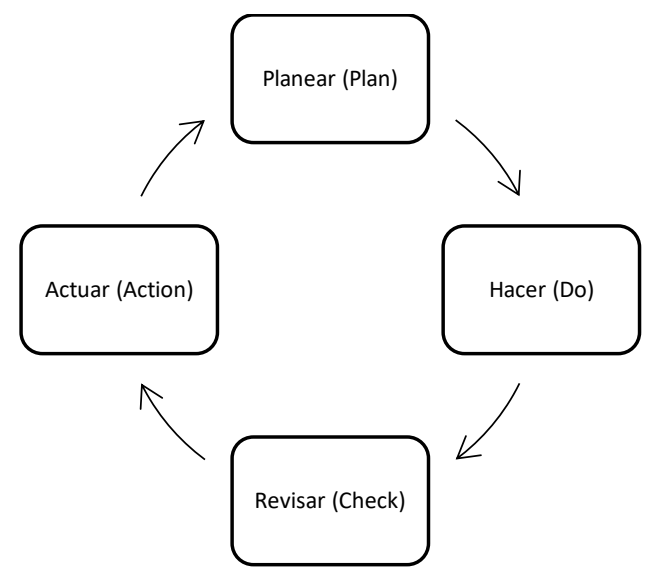

Fuente: Elaboración propia de los autores

\subsection{MOORA}

Método Multi Objetivo de Optimización basado en Análisis de Radio, MOORA (por sus siglas en inglés, Multi Objective Optimization on basis of Ratio Analysis), es un método el cual evalúa el rendimiento de varias alternativas dentro de una matriz con determinados criterios de costes y beneficios mediante la diferencia entre las sumas de sus valores normalizados (Pérez-Domínguez et al., 2015; Dwivedi et al., 2020). Dicho método clasifica todas las alternativas de acuerdo con los radios obtenidos. Para luego con dicha información determinar la alternativa que mejor cumple con los criterios establecidos. También es un método utilizado en los sistemas de apoyo para las decisiones que tienen un nivel de flexibilidad y facilidad de comprensión para separar la parte subjetiva de un proceso de evaluación con los criterios de ponderación de decisiones (Arisman et al., 2021) y además también tiene un buen nivel de selectividad porque puede determinar cuáles criterios son beneficiosos (Beneficio) o desfavorables (Costo). (Lestari et al.,2021).

Este método se basa en tres suposiciones que se deben tener en cuenta para el método: la primera es la suposición de los números cardinales, estos números son definidos por medición directa o alternativa o como números adimensionales, lo que nos permite usar las cuatro operaciones esenciales de la aritmética: sumar, restar, multiplicar y dividir (Brauers et al., 2009); el segundo son las elecciones discretas, que pueden ser casos discretos, consta de una serie de alternativas posibles y bien definidas (proyectos, diseño) o en caso continuo genera alternativas a partir de un conjunto continuo de opciones durante el propio proceso, y por último el supuesto de asumir atributos, para definir mejor los objetivos debemos centrarnos en la noción de atributo, es decir, que un objetivo y un atributo se relacionan mutuamente. (Brauers et al., 2006)

\section{Metodología}

El método se desarrolla mediante los siguientes pasos:

Paso 1. Determinar la matriz inicial de decisión. En este paso se identifican las alternativas $y$ criterios a utilizar para 
desarrollar una matriz de decisión como se observa en la Ec. (1).

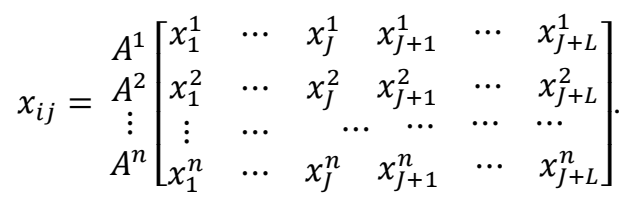

Donde:

$n$ son las filas de la matriz que se representan con las alternativas en evaluación $\left(A^{1}, A^{2}, \ldots, A^{n}\right)$.

$J+L$ son las columnas que representan los criterios de evaluación de las alternativas.

$J$ criterios cuantitativos y $L$ criterios cualitativos.

$A^{i}$ son las alternativas para $i=1,2 \ldots n$.

$x_{i j}$ representa las entradas de la alternativa i con respecto al criterio $j$.

Paso 2. Calcular la matriz de decisión normalizada. Los atributos se pueden expresar en diferentes unidades de medida, es por ello es necesario la normalización con distancia euclidiana. Como se muestra en la Ec. (2). (Brauers, 2009)

$$
N x_{i j}=\frac{x_{i j}}{\sqrt{\sum_{1}^{n} x_{i}^{2}}} .
$$

Donde cada una de las entradas $x_{i j}$ se divide entre la raíz de la suma de los cuadrados del criterio $x_{j}$. El resultado de esta operación son valores adimensionales, valores que pertenecen al intervalo $[0,1]$, que carecen de escala los cuales permiten usar las cuatro operaciones esenciales de la aritmética entre criterios. (Gadakh, 2010)

Paso 3. Calcular la matriz de decisión normalizada ponderada. Para calcular la ponderación de cada criterio es necesario utilizar la Ec. (3).

$$
W N x_{i j}=w_{i} \cdot N x_{i j} \text {. }
$$

Donde $w_{i}$ es el peso del criterio, el cual se determina por decisión del autor, aunque existen algunos métodos para determinar dicho valor como: asignación directa, entropía, comparaciones de a pares, entre otros. (PérezDomínguez et al., 2017)

Paso 4. Clasificar los criterios de costos o de beneficio de cada alternativa. Para los criterios de beneficios, que son los criterios en los que su objetivo es maximizar el resultado, se calcula como la suma de todas las calificaciones normalizadas ponderadas utilizando la Ec. (4).

$N x_{i}=w \cdot N x_{i} \mid \in J^{\max }$,

Donde $J^{\max }$ se asocia con criterios de beneficio. De esta misma manera se calcula para los criterios de costos, que son los criterios que tienen como objetivo minimizar el resultado, como se muestra en la Ec. (5)

$N x_{j}=w \cdot N x_{j} \mid \in J^{\text {min }}$.

Donde $J^{\text {min }}$ se asocia con criterios de costos.

Paso 5. Calcular la contribución de cada alternativa. Para calcular la contribución de cada alternativa se utiliza la Ec. (6).

$N y_{i}=\sum_{i=1}^{g} N x_{i}-\sum_{j=g+1}^{m} N x_{j}$.

Donde:

$N y_{i}$ es la contribución de cada alternativa $i=1,2, \ldots, n$.

$\sum_{i=1}^{g} N x_{i}$ es la sumatoria de los criterios de beneficio.

$\sum_{j=g+1}^{m} N x_{j}$ es la sumatoria de los criterios de costos.

El valor de $N y_{i}$ puede resultar en un valor negativo o positivo dependiendo de los criterios de beneficios y los criterios de costos. Una vez obtenidos los valores de $N y_{i}$ se ordenan estos resultados de mayor a menor, siendo el resultado mayor la alternativa que cumple con los requisitos anteriormente planteados. (Chérrez-Troya et al., 2018)

\subsection{Escala de Likert}

Es una escala psicométrica que puede valorar las nociones, conocimiento y aplicabilidad de determinada situación que se 
utiliza principalmente en cuestionarios para obtener el grado "de acuerdo" con una declaración o conjunto de declaraciones (Prieto et al., 2021). Dichas escalas son técnicas de escalado no comparativa y son unidimensionales, es decir, que solo miden un rasgo único. Los participantes en ese tipo de encuestas suelen responder en que grado están de acuerdo con una declaración determinada. (Jamieson, 2004)

La escala que se usa comúnmente es la escala de 5 puntos, que va desde el extremo "totalmente en desacuerdo" hasta el otro extremo "totalmente de acuerdo" y en medio "ni en desacuerdo ni de acuerdo". A cada una de estas opciones se le asigna un valor numérico que inicia desde 1 y se incrementa uno por cada nivel. Como se muestra en la Tabla 1.

TABLA 1. ESCALA LIKERT

\begin{tabular}{lllll}
\hline $\begin{array}{l}\text { Totalmen } \\
\text { te en } \\
\text { desacuer } \\
\text { do }\end{array}$ & $\begin{array}{l}\text { En } \\
\text { do }\end{array}$ & $\begin{array}{l}\text { desacuer } \\
\text { do ni de } \\
\text { acuerdo }\end{array}$ & $\begin{array}{l}\text { acuer } \\
\text { do }\end{array}$ & $\begin{array}{l}\text { Totalmen de } \\
\text { acuerdo }\end{array}$ \\
\hline 1 & 2 & 3 & 4 & 5 \\
\hline
\end{tabular}

Fuente: Elaboración propia de los autores en base a los resultados.

\subsection{Materiales y Métodos}

En esta sección se presenta el método que se utiliza para plantear la solución al problema mediante la Figura 2.

\section{CASO EXPERIMENTAL}

En esta sección se documentan los resultados y discusiones del trabajo de investigación.

Paso 1. Definición del problema

Los criterios se relacionan con los objetivos del estudio, utilizando este mismo planteamiento y los objetivos planteados en el PDCA en el artículo de Li et al. (2020) y como se muestran la Figura 3 los criterios de la matriz son los siguientes: $\rightarrow$ Atención Medica Básica $\left(C_{1}\right)$. Modificación de la cantidad de rondas médicas y organización de estudios médicos semanales para discutir diariamente la condición de los pacientes críticos.

$\rightarrow$ Personal Médico por Cama $\left(C_{2}\right)$. Según en su estudio confirmo que la cantidad de personal médico y camas disponibles en la unidad de cuidados intensivos afectará significativamente la mortalidad de los pacientes críticos. (Landrigan et al., 2004)

\section{FIGURA 2. DIAGRAMA DE FLUJO PDCA-MOORA}

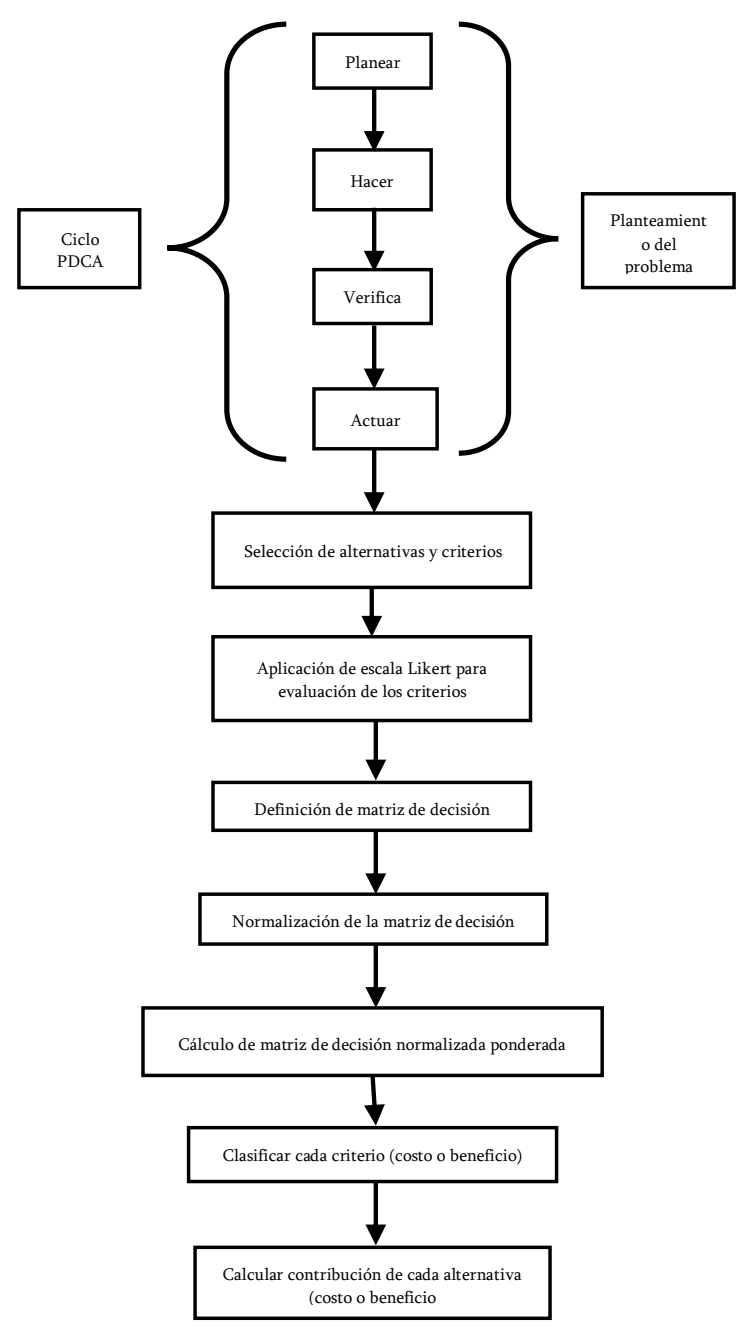

Fuente: Elaboración propia de los autores en base a los resultados. 
Aplicación del metodo moora para la gestión de la carga de trabajo en la atención de pacientes con COVID-19

$\rightarrow$ Equipos médicos intensivos $\left(C_{3}\right)$. Realización de una gestión centralizada para los equipos médicos de la unidad de cuidados intensivos.

$\rightarrow$ Tiempo de guardia médica $\left(C_{4}\right)$. Organización de turnos flexibles para garantizar la calidad médica.

$\rightarrow$ Utilización de plataformas de comunicación digitales $\left(C_{5}\right)$. Utilización de plataformas como WeChat, Whatsapp, Telegram, entre otras, para la comunicación entre la sala de aislamiento y el exterior.

\section{Alternativas}

Las alternativas se establecieron como la percepción de 3 expertos que utilizando la escala Likert realizaran una evaluación de la importancia de cada uno de los criterios establecidos anteriormente.

Experto 1

Experto 2

Experto 3

FIGURA 3. CICLO PDCA APLICADO POR LI ET AL. (2020)

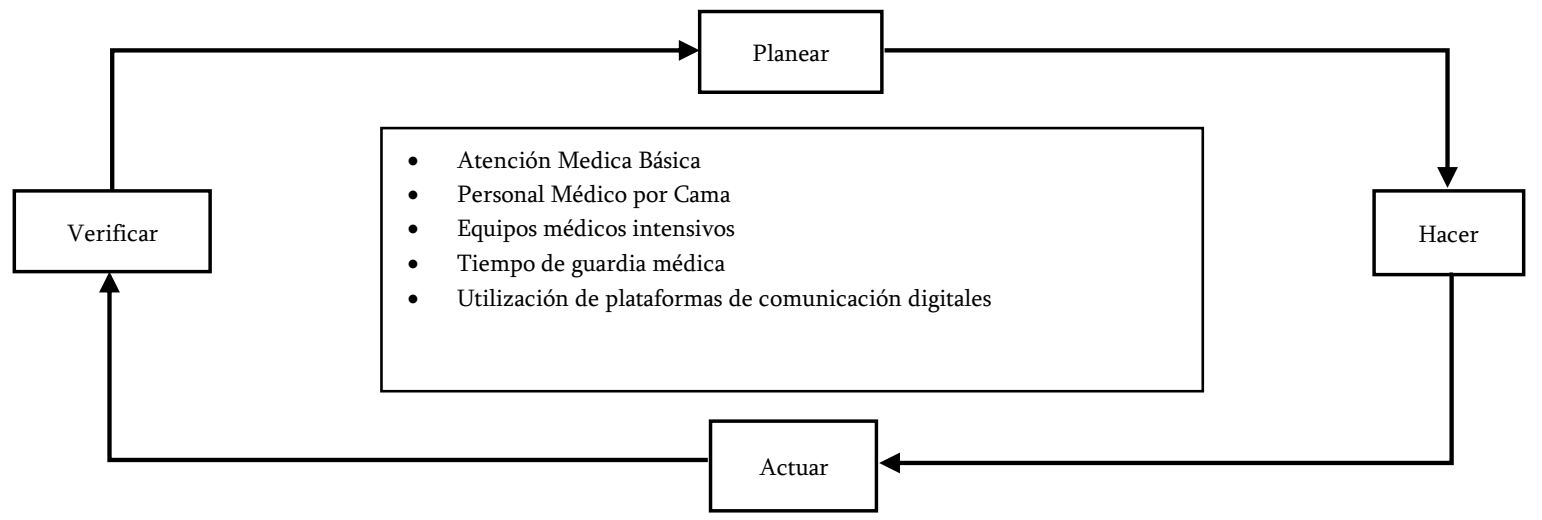

Fuente: Preparado por Li et al (2020)

Paso 2. Usar escala de Likert para obtener el grado de importancia de los criterios. Para evaluar el grado de importancia de cada uno de los criterios se usa la escala de Likert pero con modificaciones en cada uno de los niveles. Como se muestra en la Tabla 2.

TABLA 2. ESCALA LIKERT MODIFICADA

\begin{tabular}{|l|l|l|l|l|}
\hline $\begin{array}{l}\text { Sin } \\
\text { Importa } \\
\text { ncia }\end{array}$ & $\begin{array}{l}\text { De poca } \\
\text { importa } \\
\text { ncia }\end{array}$ & $\begin{array}{l}\text { Moderada } \\
\text { mente } \\
\text { importante }\end{array}$ & $\begin{array}{l}\text { Import } \\
\text { ante }\end{array}$ & $\begin{array}{l}\text { Muy } \\
\text { import } \\
\text { ante }\end{array}$ \\
\hline 1 & 2 & 3 & 4 & 5 \\
\hline
\end{tabular}

Paso 3. Matriz de decisión. En base a lo antes mencionando, utilizando la Ec. (1) y la escala de Likert con las calificaciones de los expertos la matriz de decisión inicial se definió de la siguiente manera en la Tabla 3.
TABLA 3. MATRIZ DE DECISIÓN

\begin{tabular}{|l|c|c|c|c|c|}
\cline { 2 - 6 } \multicolumn{1}{c|}{} & $\boldsymbol{C}_{\mathbf{1}}$ & $\boldsymbol{C}_{\mathbf{2}}$ & $\boldsymbol{C}_{\mathbf{3}}$ & $\boldsymbol{C}_{\mathbf{4}}$ & $\boldsymbol{C}_{\mathbf{5}}$ \\
\hline Experto 1 & 2 & 3 & 4 & 5 & 1 \\
\hline Experto 2 & 1 & 5 & 3 & 1 & 4 \\
\hline Experto 3 & 4 & 1 & 5 & 3 & 2 \\
\hline
\end{tabular}

Paso 4. Calcular la matriz de decisión normalizada. Apoyándonos en el programa Microsoft Excel se realizaron los cálculos de la matriz normalizada utilizando la Ec. (2). El resultado de la operación se muestra en la Tabla 4.

TABLA 4. MATRIZ DE DECISIÓN NORMALIZADA

\begin{tabular}{|l|c|c|c|c|c|}
\cline { 2 - 6 } \multicolumn{1}{c|}{} & $\boldsymbol{C}_{\mathbf{1}}$ & $\boldsymbol{C}_{\mathbf{2}}$ & $\boldsymbol{C}_{\mathbf{3}}$ & $\boldsymbol{C}_{\mathbf{4}}$ & $\boldsymbol{C}_{\mathbf{5}}$ \\
\hline Experto 1 & 0.436 & 0.507 & 0.566 & 0.845 & 0.218 \\
\hline Experto 2 & 0.218 & 0.845 & 0.424 & 0.169 & 0.873 \\
\hline Experto 3 & 0.873 & 0.169 & 0.707 & 0.507 & 0.436 \\
\hline
\end{tabular}


López Castañeda, J. R., Pérez-Domínguez, L. \& García Alcaraz, J. L.

Paso 5. Calcular la matriz de decisión normalizada ponderada. Para realizar esto, se definió la ponderación de cada criterio por medio de evaluación por percepción y se le asigno un valor porcentual. Como se muestra en la Tabla 5.

TABLA 5. DEFINICIÓN DE LOS PESOS DE LOS CRITERIOS

\begin{tabular}{|l|l|l|}
\cline { 2 - 3 } \multicolumn{1}{l|}{} & Descripción & Peso (\%) \\
\hline $\boldsymbol{C}_{\mathbf{1}}$ & Atención Medica Básica & 0.25 \\
\hline $\boldsymbol{C}_{\mathbf{2}}$ & Personal Médico por Cama & 0.15 \\
\hline $\boldsymbol{C}_{\mathbf{3}}$ & Equipos médicos intensivos & 0.3 \\
\hline $\boldsymbol{C}_{\mathbf{4}}$ & Tiempo de guardia médica & 0.15 \\
\hline $\boldsymbol{C}_{\mathbf{5}}$ & $\begin{array}{l}\text { Utilización de plataformas de comunicación } \\
\text { digitales }\end{array}$ & 0.15 \\
\hline
\end{tabular}

Utilizando la Ec. (3) y la tabla de pesos anterior se calculó la siguiente matriz de decisión normalizada ponderada. Como se muestra en la Tabla 6.

TABLA 6. MATRIZ NORMALIZADA PONDERADA

\begin{tabular}{|c|c|c|c|c|c|}
\cline { 2 - 6 } \multicolumn{1}{c|}{} & $\boldsymbol{C}_{1}$ & $\boldsymbol{C}_{2}$ & $\boldsymbol{C}_{3}$ & $\boldsymbol{C}_{4}$ & $\boldsymbol{C}_{5}$ \\
\hline Experto 1 & 0.109 & 0.076 & 0.170 & 0.127 & 0.033 \\
\hline
\end{tabular}

\begin{tabular}{|l|l|l|l|l|l|}
\hline Experto 2 & 0.055 & 0.127 & 0.127 & 0.025 & 0.131 \\
\hline Experto 3 & 0.218 & 0.025 & 0.212 & 0.076 & 0.065 \\
\hline
\end{tabular}

Paso 5. Clasificar los criterios de costos o de beneficio de cada alternativa. En la Tabla 7 se asigna si el criterio es de costo $\left(J^{\mathrm{min}}\right)$ o beneficio $\left(J^{\max }\right)$. Como se plantan en las Ec. (4) y (5).

TABLA 7. ASIGNACIÓN DE COSTO O BENEFICIO A CADA CRITERIO

\begin{tabular}{|l|l|l|}
\cline { 2 - 3 } \multicolumn{1}{c|}{} & Descripción & $\begin{array}{l}\text { Tipo de } \\
\text { criterio }\end{array}$ \\
\hline $\boldsymbol{C}_{\mathbf{1}}$ & Atención Medica Básica & Beneficio \\
\hline $\boldsymbol{C}_{2}$ & Personal Médico por Cama & Costo \\
\hline $\boldsymbol{C}_{3}$ & Equipos médicos intensivos & Beneficio \\
\hline $\boldsymbol{C}_{4}$ & Tiempo de guardia médica & Costo \\
\hline $\boldsymbol{C}_{\mathbf{5}}$ & $\begin{array}{l}\text { Utilización de plataformas de } \\
\text { comunicación digitales }\end{array}$ & Costo \\
\hline
\end{tabular}

Paso 6. Calcular la contribución de cada alternativa. Utilizando la Ec. (6) se calcula el valor de $N y_{i}$ y se realiza el ranqueo de los criterios ordenando los valores del mayor al menor siendo el primero con el primero con el $N y_{i}$ más alto. Como se muestra en la Tabla 8.

TABLA 8. CONTRIBUCIÓN DE CADA ALTERNATIVA

\begin{tabular}{|l|l|l|l|l|l|l|}
\cline { 3 - 7 } \multicolumn{2}{c|}{} & Experto 1 & Experto 2 & Experto 3 & \multicolumn{1}{c|}{$\boldsymbol{N y}_{\boldsymbol{i}}$} & Ranking \\
\hline Beneficio & $\boldsymbol{C}_{\mathbf{1}}$ & 0.109 & 0.055 & 0.218 & -0.304 & 5 \\
\hline Costo & $\boldsymbol{C}_{\mathbf{2}}$ & 0.076 & 0.127 & 0.025 & 0.228 & 3 \\
\hline Beneficio & $\boldsymbol{C}_{\mathbf{3}}$ & 0.170 & 0.127 & 0.212 & -0.176 & 4 \\
\hline Costo & $\boldsymbol{C}_{\mathbf{4}}$ & 0.127 & 0.025 & 0.076 & 0.228 & 2 \\
\hline Costo & $\boldsymbol{C}_{\mathbf{5}}$ & 0.033 & 0.131 & 0.065 & 0.229 & 1 \\
\hline
\end{tabular}

Por los resultados obtenidos el criterio que se debe enfocar el área de cuidados intensivos es en el Criterio $5\left(C_{5}\right)$ que es utilizar las plataformas de comunicación digitales para mejorar la comunicación entre la sala de aislamiento y el exterior. Aunque por los resultados tan equitativos también se le tiene que dar enfoque a los Criterios $2\left(C_{2}\right)$ y $4\left(C_{4}\right)$ que son el personal médico por cama y el tiempo de guardia médica respectivamente. Esto debido a que la diferencia no muy grande ( 1 milésima de unidad) entre los Criterios 5, 4 y 2, como se muestra en los resultados se tiene que enfocar la prioridad en el Criterio 5, también se debería empezar a trabajar en los Criterios 2 y 4 . Estos resultados se pueden mejorar, si los expertos son los médicos del área de cuidados intensivos, pero por la situación actual de la pandemia y el poco tiempo no se utilizó ese recurso.

\section{Resultados}

Para el análisis de resultados se utilizó el análisis de correlación de Pearson, los datos de la Tabla 6 son los que se utilizaron para el análisis de correlación obteniendo los resultados que se muestra la Figura 4 y Tabla 9. 
Aplicación del metodo moora para la gestión de la carga de trabajo en la atención de pacientes con COVID-19

FiguRA 4. GRAFICA DEL ANÁLISIS DE CORRELACIÓN DE PEARSON

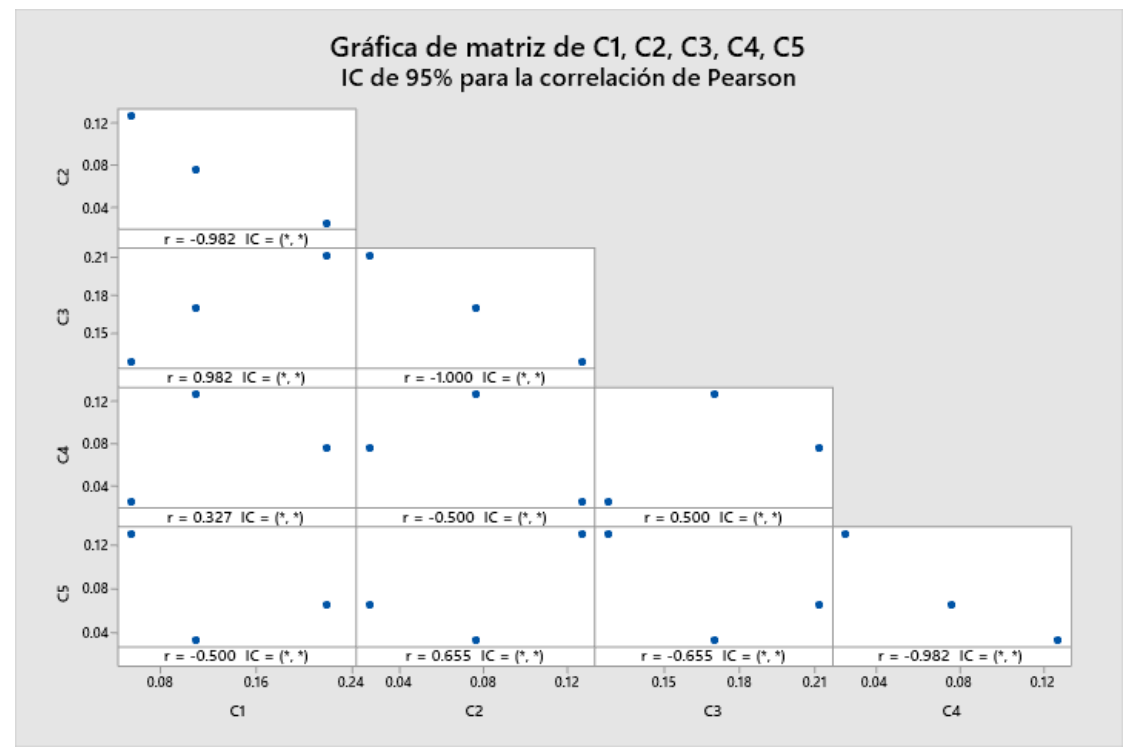

Fuente: Elaboración propia de los autores en base a los resultados.

TABLL 9. TABLA DEL ANÁLISIS DE CORRELACIÓN DE PEARSON

\begin{tabular}{|l|l|l|l|l|}
\hline & $C 1$ & $C 2$ & $C 3$ & $C 4$ \\
\hline$C 2$ & -0.982 & & & \\
\hline$C 3$ & 0.982 & -1.000 & & \\
\hline$C 4$ & 0.327 & -0.500 & 0.500 & \\
\hline$C 5$ & -0.500 & 0.655 & -0.655 & -0.982 \\
\hline
\end{tabular}

Por los resultados obtenidos, la relación entre $C_{1}$ y $C_{2}$ es de -0.982 lo que significa que la atención medica básica no afecta la cantidad de personal medico por cama. La relación entre $C_{1}$ y $C_{3}$ es de 0.982 lo que significa que la atención medica básica afecta a la cantidad de equipos médicos intensivos. La relación entre $C_{1}$ y $C_{4}$ es de 0.327 lo que significa que la atención medica afecta medianamente afecta al tiempo de guardia médica. La relación entre $C_{1}$ y $C_{5}$ es de -0.500 lo que significa que la atención medica no afecta a la utilización de plataformas digitales para la comunicación de la sala de aislamiento con el exterior. La relación entre $C_{2}$ y $C_{3}$ es de -1.000 lo que significa que la cantidad de personal medico por cama no afecta a la cantidad de equipos médicos intensivos. La relación entre $C_{2}$ y $C_{4}$ es de -0.500 lo que significa que la cantidad de personal médico por cama no afecta al tiempo de guardia médica. La relación entre $C_{2}$ y $C_{5}$ es de 0.655 lo que significa que la cantidad de personal medico por cama afecta a la utilización de plataformas digitales para la comunicación de la sala de aislamiento con el exterior. La relación entre $C_{3}$ y $C_{4}$ es de 0.500 lo que significa que la cantidad de equipos médicos intensivos afecta al tiempo de guardia médica. La relación entre $C_{3}$ y $C_{5}$ es de -0.655 lo que significa que la cantidad de equipos médicos intensivos no afecta a la utilización de plataformas digitales para la comunicación de la sala de aislamiento con el exterior. $\mathrm{Y}$ por último la relación entre $C_{4}$ y $C_{5}$ es de -0.982 lo que significa que la cantidad de equipos médicos intensivos no afecta a la utilización de plataformas digitales para la comunicación de la sala de aislamiento con el exterior. Retomando los resultados del método MOORA y los resultados del análisis de correlación de Pearson si el área de cuidados intensivos se enfoca en la utilización de plataformas digitales para la comunicación de la sala de aislamiento con el exterior afectaría a la cantidad de personal médico esto reforzaría el resultado del método MOORA. 


\section{CONCLUSIONES}

El sistema de atención medica es de suma importancia a la hora de atender pacientes graves de COVID-19. Por los resultados obtenidos del método MOORA el criterio el criterio al que hay que implementar es el de utilizar plataformas de comunicación digitales aunque, considerando los resultados del análisis de Pearson, hay que tener en cuenta que estaríamos afectando la cantidad de personal médico, y teniendo en cuenta los demás criterios que se evaluaron considero que es lo primero que se tiene que realizar en estos casos de emergencia. Porque las plataformas de mensajería como WeChat, WhatsApp, Telegram ofrecen una comunicación instantánea por medio de texto, imágenes, documentos, videos o audios y que no requiere presencia física entre la sala de aislamiento y el exterior, pero si requieren una conexión a internet estable. Aunque si comparamos estos resultados con los del PDCA, el método MOORA definirá el orden en que deben realizarse cada uno de los criterios planteados para lograr una óptima atención medica de los pacientes graves por COVID-19. Se pueden obtener mejores resultados si los expertos se sustituyeran por doctores especializados en el tema y si se realizara una encuesta, pero esto no fue posible debido a las limitaciones actuales por la pandemia. El método MOORA, así como otros métodos multicriterio tienen diversas aplicaciones y esperemos que esta información también sirva como referencia a futuras aplicaciones..

Contribuciones de los autores: Conceptualización; Jesus Raymundo Lopez Castañeda y Luis Pérez Domínguez, Metodología; Luis Pérez y Dr. Jorge Luis García Alcaraz Validación; Jesús Raymundo López Castañeda, Luis Pérez Domínguez, Análisis formal; Jesús Raymundo López Castañeda, Luis Pérez Domínguez, redacción-borrador original; Jesús Raymundo López Castañeda, redacción-revisión y edición. Jesús Raymundo
López Castañeda, Luis Pérez Domínguez y Dr. Jorge Luis García Alcaraz. Todos los autores han leído y aceptado la versión publicada del manuscrito".

Financiación: Esta investigación no recibió financiación externa.

Declaración de disponibilidad de datos: Esencialmente los datos usados para plantear el caso de estudio fueron tomados por el reporte publicado por Li, Wang, \& Jiao (2020). De tal modo que fue posible demostrar la validez de la metodología aquí propuesta. La contribución del presente documento es la aplicación de método multicriterio MOORA para analizar un escenario COVID bajo un ambiente teórico.

Agradecimientos: Sin agradecimientos.

Conflictos de intereses: "Los autores declaran no tener ningún conflicto de intereses".

\section{REFERENCIAS}

Arisman, A., \& Sianturi, F. A. (2021). Sistem Pendukung Keputusan Penerimaan Siswa Baru Menggunakan Metode Moora (Multi-Objective Optimization On The Basis Of Ratio Analysis). Jurnal Ilmu Komputer dan Sistem Informasi (JIKOMSI), 3(3), 73-83.

Brauers, W. K., \& Zavadskas, E. K. (2006). The MOORA method and its application to privatization in a transition economy. Control and cybernetics, 35, 445-469.

Brauers, W. K., \& Zavadskas, E. K. (2009). Robustness of the multi-objective MOORA method with a test for the facilities sector. Technological and economic development of economy, 15(2), 352-375.

Chérrez-Troya, M., Martínez-Gómez, J., Peralta-Zurita, D., \& Antonio Llanes-Cedeño, E. (2018). Métodos multicriterio aplicados en la selección de un material para discos de freno. Ingenius. Revista de Ciencia y Tecnología, (20), 8395.

Dwivedi, R., Kumar, S., Gupta, N., \& Prasad, K. (2020, November). Application of MOORA method to analyze the factors affecting success of social programs. In AIP Conference Proceedings (Vol. 2273, No. 1, p. 050058). AIP Publishing LLC.

Gadakh, V. S. (2010). Application of MOORA method for parametric optimization of milling process. International Journal of Applied Engineering Research, 1(4), 743.

Li, Y., Wang, H., \& Jiao, J. (2020). The application of strong matrix management and PDCA cycle in the management of severe COVID-19 patients. 
Pérez-Domínguez, L., Alvarado-Iniesta, A., RodríguezBorbón, I., \& Vergara-Villegas, O. (2015). Intuitionistic fuzzy MOORA for supplier selection. Dyna, 82(191), 3441.

Jamieson, S. (2004). Likert scales: How to (ab) use them?. Medical education, 38(12), 1217-1218.

Johnson, C. N. (2002). The benefits of PDCA. Quality Progress, 35(5), 120.

Kalibatas, D., \& Turskis, Z. (2008). Multicriteria evaluation of inner climate by using MOORA method. Information technology and control, 37(1).

Landrigan, C. P., Rothschild, J. M., Cronin, J. W., Kaushal, R., Burdick, E., Katz, J. T., ... \& Czeisler, C. A. (2004). Effect of reducing interns' work hours on serious medical errors in intensive care units. New England Journal of Medicine, 351(18), 1838-1848.

Lestari, N., Karman, J., \& Santoso, B. (2021). Komparasi Metode Weighted Aggregated Sum Product Assessment (WASPAS) dan Multi-Objective Optimization on The Basis of Ratio (MOORA) Dalam Penerimaan Dosen. Journal of Information System Research (JOSH), 2(2), 138-147.

Pérez-Domínguez, L., Macías-García, J. L., Sánchez-Mojica, K. Y., \& Luviano-Cruz, D. (2017). Comparación Método multi-criterio TOPSIS y MOORA para la optimización de un proceso de inyección de plástico. Mundo FESC, 7(14), 98-105.

Prieto, A. G., \& Gómez, M. I. J. (2021). Proceso de elaboración y validación de cuestionario Likert:"Utilidad y aplicación de los diagnósticos de enfermería en la práctica clínica". Conocimiento Enfermero, 4(11), 1-12. 